Original Article

\title{
ROLE OF BEVACIZUMAB FOR PREVENTION OF NEOVASCULAR GLAUCOMA IN ACUTE RETINAL VEIN OCCLUSION
}

\author{
Bassam Mubarik*, Adeen Akram** \\ *Assistant Professor, Department of Ophthalmology Madinah Teaching Hospital Faisalabad \\ ${ }^{* *}$ House Officer, Allied Hospital, Faisalabad
}

\begin{abstract}
:
There is a possibility of developing neovascular glaucoma in ischemic type of retinal vein occlusion. Purpose of the study is to determine the role of anti VEGF agent bevacizumab for prevention of neovascular glaucoma in ischemic type of retinal vein occlusion.

METHOD: It was a retrospective study of 34 cases with unilateral central retinal vein occlusion. All of them were treated with intra vitreous Bevacizumab for management of associated macular edema. Primary outcome was to study development of neovascular glaucoma in these cases.

RESULTS: Thirty four cases were selected with retinal vein occlusion. Seventeen cases were of non ischemic type and remaining 17 were of ischemic type vein occlusion. During a follow up period of two years non ischemic group was given on average 8 bevacizumab injection. Only one of these non ischemic eyes developed neo vessels of optic disc but neo vascular glaucoma did not develop. In case of ischemic group during follow up period of two years a mean of 6 bevacizumab injections were given. In this ischemic group 2 cases developed iris neovesseles and 3 cases developed retinal neovesseles. Out of these, three eyes progressed to neovascular glaucoma.
\end{abstract}

CONCLUSION: Bevacizumab treatment of macular edema for ischemic central retinal vein occlusion does not prevent neovascular glaucoma.

\section{INTRODUCTION:}

Central retinal vein occlusion is one of the most common causes of sudden loss of vision. It is second only to diabetes in causing retinal loss of vision. ${ }^{[1]}$ There are two types of central retinal vein occlusion ischemic and non ischemic. Out of these cases, $20 \%$ are of ischemic type and $80 \%$ are non ischemic. ${ }^{[2,3]}$ Ischemic central retinal vein occlusion can lead to neo vascular glaucoma with an incidence of $23 \%$ to $60 \% .^{\left[{ }^{4]}\right.}$ According to central retinal vein occlusion study, prophylactic Pan Retinal Photocoagulation does not prevent development of neovascular glaucoma. ${ }^{2}$ Neovascular glaucoma that occurs after this kind of retinal vein occlusion is also called as "hundred day glaucoma".
Development of neovesseles in case of retinal vein occlusion is due to the ischemia of involved area of retina. In this regard, release of vascular endothelial growth factor (VEGF) from ischemic area stimulates neovessel formation from vascular tissue. ${ }^{[5,6]}$ Bevacizumab is an anti-VEGF agent. It has a proven action for prevention of neovascular tissue formation in central retinal vein occlusion and can be used as a treatment option along with other treatment options for neovascular glaucoma of ischemic retinal vein occlusion. ${ }^{[7,8]}$ During past few years anti VEGF agents are being used successfully for macular

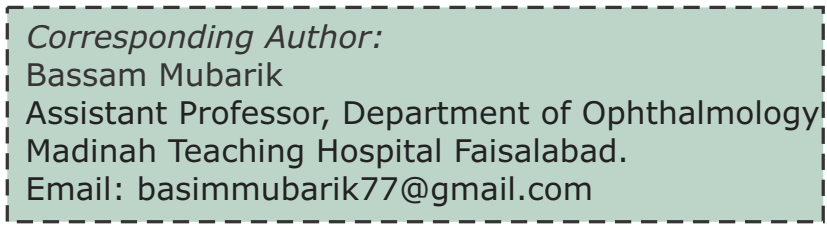


edema that develops in these retinal vein occlusion cases. ${ }^{[9,10,11]}$

This study investigates those cases of ischemic and non ischemic retinal vein occlusion which were treated with anti VEGF agent, Bevacizumab for macular edema component of disease, for protective effect of this agent against development of neovascular glaucoma in central retinal vein occlusion.

\section{MATERIAL AND METHOD:}

Study was conducted at Ophthalmology Department, Madina Teaching Hospital, Faisalabad. It was a retrospective study. All those cases were reviewed which were treated with anti-VEGF agent, Bevacizumab for macular edema component of central retinal vein occlusion. All these patients were under treatment of our retina clinic during March 2011 to August 2015.

Patients with macular edema due to central retinal vein occlusion with onset of disease within three months were included in the study, both ischemic and non ischemic type. Patients with neovesseles or neo vascular glaucoma already present and patients with history of proliferative diabetic retinopathy were excluded from the study. Patients were labeled as ischemic or non ischemic by retina specialist with the help of Visual acuity examination, pupillary examination and non perfusion areas on angiography. Macular edema was diagnosed with the help of OCT. The primary outcome was development of neovascular glaucoma in ischemic vein occlusion or non ischemic vein occlusion even with treatment with bevacizumab. Secondary outcome measure was formation of ocular neovesseles without development of neovascular glaucoma.

\section{RESULTS:}

This study included 34 eyes of 34 patients with a diagnosis of central retinal vein occlusion (CRVO). Seventeen eyes of 17 patients had ischemic central retinal vein occlusion and 17 eyes of 17 patients had non ischemic central retinal vein occlusion. Average age at presentation was 71 years in ischemic group and 69.5 years in non ischemic group. All 34 eyes received intra vitreal bevacizumab injection. Mean follow up duration was 23 months for ischemic group and 24 months for non ischemic group. Ischemic eyes received an average of 6 injections during follow up period and non ischemic eyes received an average of 8 injections during follow up period. Ocular neo vessels developed in one of 17 eyes in non ischemic group and 5 of 17 in ischemic group. Out of these 5 eyes, 2 eyes developed neovesseles of iris, 2 eyes developed neovesseles of optic disc and one eye developed vitreous hemorrhage. Four of these eyes also received pan retinal photocoagulation and one could not be given pan retinal photocoagulation due to thick vitreous hemorrhage. Both of the eyes with neovesseles of iris developed neovascular glaucoma despite that pan retinal photocoagulation was applied. One of the eyes with vitreous hemorrhage also developed neo vascular glaucoma. Thus 3 of 17 ischemic eyes developed neovascular glaucoma. Regarding non ischemic retinal vein occlusion only one out of 17eyes developed neo vessels which did not progress to neo vascular glaucoma.

Data was statistically analyzed to compare the relative occurance of neo vascular glaucoma between two groups and showed non significant difference between ischemic and non ischemic patients included in this study.

Table No 1. Baseline features of patients with central retinal vein occlusion

\begin{tabular}{|l|l|l|}
\hline Characteristics & Non ischemic CRVO & Ischemic CRVO \\
\hline No. of patients & 17 & 17 \\
\hline Average age & 69.5 & 71 \\
\hline Sex male/female & $11 / 6$ & $11 / 6$ \\
\hline Average follow up period & 24 months & 23 months \\
\hline
\end{tabular}


Table No 2. Clinical features of patients with central retinal vein occlusion.

\begin{tabular}{|l|l|l|}
\hline Clinical feature & Non ischemic CRVO & Ischemic CRVO \\
\hline Average no. of injections & 06 & 08 \\
\hline Retinal neo vessels & 01 patient & 05 patients \\
\hline Iris neo vessels & 0 patients & 02 patients \\
\hline Vitreous hemorrhage & 0 patients & 01 patients \\
\hline Neo vascular glaucoma & 0 patients & 03 patients \\
\hline
\end{tabular}

\section{DISCUSSION:}

Ischemic central retinal vein occlusion is associated with a high rate of neovascular process in eye and if it is not properly addressed it will lead to neovascular glaucoma in up to $23 \%$ to $60 \%$ of cases. ${ }^{12}$ It has been studied that during first six months of presentation, chances of anterior segment neo vessels is very high among ischemic vein occlusion cases. ${ }^{20}$ The development of neovascular process is most probably associated with severity of retinal ischemia. ${ }^{6}$ The neovascular glaucoma which is associated with ischemic retinal vein occlusion is also called as 100 day glaucoma which means that there is high chances of glaucoma development around this time after initial presentation of disease.

Central vein occlusion study $\mathrm{CVOS}^{2}$ was conducted in 1997. According to this study there was a high rate of early iris neo vessels in ischemic occlusion cases. Among the larger group of patients during CVOS study found that $35 \%$ of all 117 ischemic eyes developed angle or iris neo vessels and pan retinal photocoagulation was applied for its treatment but 10 of these eyes still suffered from glaucoma. After CVOS study standard management for ischemic occlusion is observation for neo vessels and application of pan retinal photocoagulation if they occur.

CRUISE study ${ }^{10}$ found that macular edema improved after anti VEGF agent Ranibizumab in central retinal vein occlusion study. The cruise sham group of 129 eyes had 11 eyes which developed neovesseles of iris during first year of study and 2 eyes suffered from glaucoma during first 6 months. In the treatment group with Ranibizumab 7 out of 261 eyes suffered from neovesseles of iris and one eye suffered from glaucoma during first year of study. These patients received on average 9.2 treatments during one year time period. However CRUISE study excluded cases with brisk relative afferent pupillary defect reaction so cases with severe ischemia were not included.

Another 2012 prospective randomized study ${ }^{21}$ of 60 central retinal vein occlusion eyes studied sham or bevacizumab injections every 6 weeks for the initial 6 months of study. Patients with very low vision up to 20/500 were included in the study. So it was likely that ischemic cases were included. Sham group consisted of 30 eyes, out of which 5 eyes suffered neovesseles of iris by 24 weeks follow up while none suffered in bevacizumab group. After week 24 all of these patients were given bevacizumab every 1.5 months. There was regression of NVI in 5 patients and no patient in study suffered glaucoma. This observation is suggestive of protection provided by anti VEGF against neovascular complications. Authors of this study suggested that anti VEGF simply delay the onset of neovesseles. The study consisted of only 12 months follow up period and ischemic or non ischemic cases were not separated.

In this study anti VEGF treatment on ischemic occlusion is similar to Hayreh's natural history study ${ }^{19}$ because separation of ischemic and non ischemic cases was performed. The cruise trial did not separate ischemic from non ischemic cases and probably ischemic cases were not included which is group of interest in the current study. Patients were selected for study within three months of follow up which is similar to Hayreh study. Follow up period was also similar to Hayreh study. Hayreh and Zimmerman also found that in ischemic vein occlusion cases there was $10 \%$ risk of developing neovesseles of optic disc during one year follow up. In current study posterior segment neovascularization was more than this study but cause is not clear.

Small sample size of 17 ischemic eyes is a limiting factor for this study. Patients were treated with different treatments according to situation demands and same treatment regimen could not be given. Gonioscopy was not performed on routine basis so cases with only angle neovesseles could be missed. Patients who received intra vitreous triamcinolone could not be excluded which could interfere with action of anti VEGF agent. Although the score study stated that triamcinolone does not interfere with rate of neovesseles of iris and neo 
vascular glaucoma but effect of triamcinolone on anti VEGF agents is yet not fully determined. . $^{13,14,15 .}$

The CRUISE study demonstrates that anti VEGF agents reduce both ocular neovascularization and neovascular glaucoma. This study also proves that anti VEGF delays neovascular events but at the end does not eliminate them.

\section{CONCLUSION:}

Anti VEGF Bevacizumab does not prevent neovascular glaucoma in ischemic vein occlusion cases when given for macular edema of these cases but it can delay it.

\section{REFERENCES:}

1. Caugati $s$, Wang $j \mathrm{j}$, Knudtnon Md, et al. Retinal vein occlusion and vascular mortality: pooled data analysis of 2 population based cohorts. Ophthalmology 2007; 114:520-524.

2. J.c.clarhson, Natural history and clinical management of central vein occlusion. Archives of ophthalmology 1997; 115: 486-491.

3. S.S. Hayreh, M.R. Klugman, M. Beri, A.E. Kimura, P. Podhajsky. Differentiation of ischemic from non ischemic central retinal vein occlusion during early acute phase. Graefe's Archive of clinical and experimental ophthalmology, 1996; 228: 201-217.

4. R.I.Meintash, S. L. Roger, L.lim et al. natural history of retinal vein occlusion: Evidence based systemic review. Ophthalmology 2010; 117: 1113-1123.

5. L.P. Aiello, R.L. Avery, P.G. Arrigg et al. vascular endothelial growth factor in ocular of patients with diabetic retinopathy and other retinal disorders. New England journal of medicine. 1994; 331:22:14801487.

6. L.P. Aiello, R.L. Avery, P.G. Arrigg et al. Vascular endothelial growth factor in ocular fluid of patients with diabetic retinopathy and other retinal disorders. New England journal of medicine.1994; 331:22:14801487.

7. M.E. Ilieve, D.doming, U-wolf-Schnurr bursh, S.wolf, and G.M. Sarra, intravitreal bevacizumab in the treatment of neovascular glaucoma. American journal of ophthalmology. 2006; 142:1054-56.
8. J. Beutel, S.Peters, M. Luke et al. Bevacizumab as adjuvant for neovascular glaucoma. Acta ophthalmologica.2010; 88: 103-109.

9. P.A. Campochiaro, j.S. Heier, L. Feiner et al.Ranibizumab for macular edema following branch retinal vein occlusion. Ophthalmology. 2010; 117:1102-1112.

10. D.M. Brown, P.A. campochiaro, R.P. Singh et al. Ranibizumab for macular edema following central retinal vein occlusion. Ophthalmology 2110; 117: 1124- 1133.

11. Beutel,F. Ziemssen, M. Luke, M. Partsch K.u. Bartz Schmidth, F. Gelisken, intravitreal bevacizumab treatment of macular edema in central retinal vein occlusion : one year results. International ophthalmology 2010; 30: 15-22.

12. S.S. Hayresh and M.B Zimmerman, Ocular neovascularization associated with central and hemi central retinal vein occlusion. Retina 2012; 32: 15531565.

13. C.K.Chan, M.S. I. P., P.C. Vaneldhuisen et al. Score study report no. 11: incidence of neovascular events in eyes with retinal vein occlusion, Ophthalmology 2011; 118: 1364-1372.

14. H.J. Sohn, D.H.Han, D.Y. Lee, changes in aqueous cytokines after intravitreous triamcinolone versus bevacizumab for macular edema in branch retinal occlusion. Acta Ophthalmologica 2013.

15. H. Noma, H. Funatsu and T. Mimura. Changes of inflammatory factors after intravitreous triamcinolone acetonoid for macular edema with central retinal vein occlusion. Journal of ocular pharmacology and therapeutics 2013; 29: 363-365.

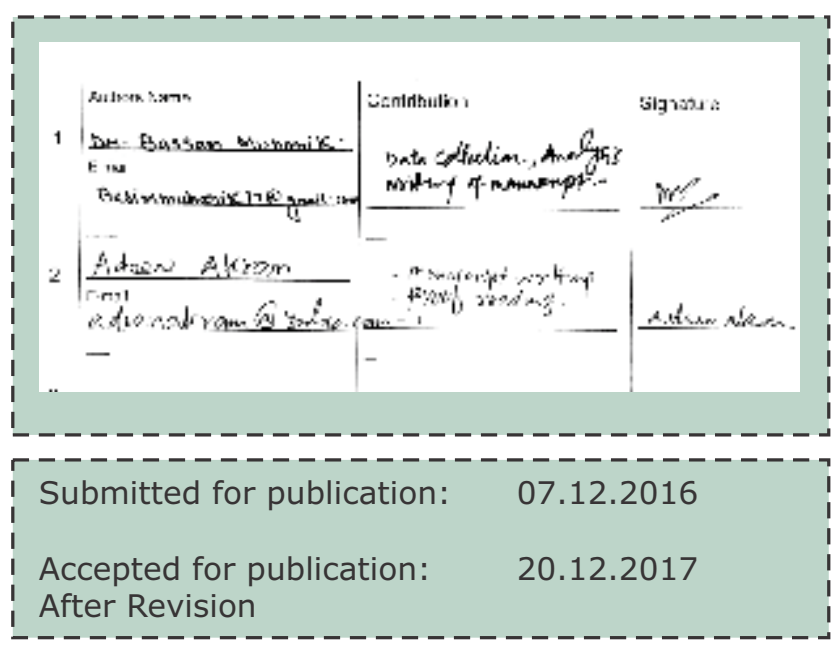

